\title{
Adult-onset Still's disease as a mask of Hodgkin lymphoma
}

\section{Ewa Dudziec, Katarzyna Pawlak-Buś, Piotr Leszczyński}

Department of Rheumatology and Rehabilitation, Poznan University of Medical Sciences, Poznan, Poland and Division

of Rheumatology and Osteoporosis, Jozef Strus Hospital, Poznan, Poland

\begin{abstract}
Adult-onset Still's disease is a rare disorder, which creates difficulties in making a proper diagnosis. Ambiguous symptoms and results of auxiliary tests, lack of unequivocal diagnostic tests and the need to exclude other causes of the disease are major problems in clinical practice.

A case of a 22-year-old woman with dominated recurrent fever, significantly elevated inflammation markers and arthritis is presented. Based on clinical signs after exclusion of infection, hematological and other reasons, the patient was diagnosed with adult-onset Still's disease. Standard treatment, with high doses of glucocorticoids and a disease-modifying drug, was applied, without the anticipated effects. The diagnostic tests were conducted again due to the lack of clinical improvement, increase of inflammatory markers and unusual response to treatment. A new symptom of significance, i.e. mediastinal lymphadenopathy, was found. After the histopathological examination of lymph nodes, Hodgkin's disease was diagnosed and targeted therapy for hematological malignancy was applied.
\end{abstract}

Key words: Hodgkin lymphoma, adult-onset Still's disease, fever of unknown etiology.

\section{Introduction}

Adult-onset Still's disease (AOSD) is a systemic inflammatory process whose etiology remains unknown. Due to its clinical presentation, the diagnosis is made after other diseases are excluded, particularly of infectious, neoplastic or hematologic etiology. The disease symptoms include high fever, rash appearing at the height of the fever, and, in nearly all patients, arthritis accompanied by muscle ache. Additionally, symptoms may also include enlargement of lymph nodes, liver or spleen. Often pneumonia, myocarditis or pericarditis occurs, confirming the systemic nature of this disease, but also indicating the severe and life-threatening course of the disease. Leukocytosis and very high ferritin levels combined with clinical symptoms indicate the diagnosis, but they are not pathognomonic for this condition. A correct diagnosis, currently based solely on classification criteria, and initiation of correct treatment are often effective in a relatively short time, which is confirmed by both clinical and laboratory tests. A phenomenal reaction to corticosteroids, nonsteroidal anti-inflammatory drugs or to treatment with methotrexate is observed. Sometimes, biological therapy with monoclonal antibodies (anti-TNF- $\alpha$, anti-IL-1 or even anti-IL-6) is required. The atypical course of the disease should increase vigilance of a rheumatology specialist and encourage verification of the diagnosis after consultation with other specialists.

\section{Case report}

A 22-year-old female patient, diagnosed two months earlier with AOSD by a rheumatologist, was admitted to the division of rheumatology for persisting clinical symptoms, in particular persistent fever and high laboratory parameters of an inflammatory reaction.

The initial problems started 10 months earlier in the form of a recurring fever, without signs of infection, or pain and swelling of joints. The patient was treated in another hospital rheumatology centre, and received

Address for correspondence:

Ewa Dudziec, Department of Rheumatology and Rehabilitation, Poznan University of Medical Sciences, Poznan, Poland and Division of Rheumatology and Osteoporosis, Jozef Strus Hospital, Poznan, Poland

Submitted: 18.03.2015; Accepted: 27.04.2015 
the initial treatment there. At that centre, significantly increased laboratory inflammatory parameters including the erythrocyte sedimentation rate (ERS), C-reactive protein (CRP), leukocytosis, anemia and thrombocythemia were observed. Diagnostic imaging procedures performed afterwards, such as posteroanterior and lateral X-ray and abdominal ultrasound, showed no anomalies.

When other disease causes were excluded and AOSD was diagnosed, treatment with methylprednisolone was initiated in i.v. pulse therapy, followed by methotrexate (MTX) with cyclosporine (CS) in combination therapy. The patient received these medications for a very short time and then discontinued the proposed therapy at her own discretion.

Two months after AOSD diagnosis, due to recurrence of symptoms, the patient was admitted to the hospital again. On admittance, recurring fever, particularly in the evenings, asthenia, muscle and joint pains, swelling of ankles, paleness of skin and tachycardia were observed. In the performed laboratory tests, high inflammatory activity was observed, together with leukocytosis and granulocytosis, anemia, thrombocythaemia, very high ferritin levels, increased liver tests and hypoalbuminemia with increased $\alpha 2$ and $\gamma$ globulin levels in the electrophoretic separation (Table I). In auxiliary tests, an enlarged heart shape in the X-ray scan of the chest and a small amount of fluid in the pericardial sac in the electrocardiograph scan were observed.

On the basis of the complete clinical image and considering Yamaguchi classification criteria (Table II), a di-

Table I. Baseline laboratory tests in a studied patient

\begin{tabular}{|lcc|}
\hline Tests & Value & Norm \\
\hline ESR $[\mathrm{mm} / \mathrm{h}]$ & 119 & $3-15$ \\
\hline CRP $[\mathrm{mg} / \mathrm{l}]$ & 303 & $0-5$ \\
\hline white blood cells $\left[10^{3} / \mathrm{mm}^{3}\right]$ & 18 & $4-10$ \\
\hline granulocytes [\%] & 86 & $40-75$ \\
\hline platelets $\left[10^{3} / \mathrm{mm}^{3}\right]$ & 819 & $140-440$ \\
\hline hemoglobin [mmol/l] & 5.3 & $7.4-9.9$ \\
\hline MCV [fl] & 73 & $80-100$ \\
\hline ferritin [ng/ml] & 1952 & $13-150$ \\
\hline ALT [U/l] & 50 & $2-33$ \\
\hline GGT [U/l] & 94 & $5-36$ \\
\hline & Proteinogram & $6-8$ \\
\hline total protein [g/dl] & 8.44 & $53-66$ \\
\hline albumin [\%] & 28 & $2-5.5$ \\
\hline$\alpha{ }_{1}$-globulin [\%] & 7.9 & $6-12$ \\
\hline$\alpha{ }_{2}$-globulin [\%] & 17 & \\
\hline$\gamma$-globulin [\%] & 27.3 & \\
\hline
\end{tabular}

Table II. Classification criteria of adult-onset Still's disease [Yamaguchi M et al., 1992] (bold criteria satisfied in the case described)

\begin{tabular}{|l|}
\hline \multicolumn{1}{|c|}{ Major criteria } \\
\hline 1) fever $39^{\circ} \mathrm{C}$ intermittent, $\geq 1$ week \\
\hline 2) arthralgia $\geq 2$ weeks \\
\hline 3) characteristic rash \\
\hline 4) white blood cells $\geq 10$ o00/ $\mu$ l with $>80 \%$ granulocytes \\
\hline 1) sore throat \\
\hline 2) lymphadenopathy \\
\hline 3) liver function tests abnormal \\
\hline 4) negative ANA and RF (anti-nuclear antibody and rheu- \\
matic factor) \\
\hline Exclusion criteria \\
\hline 1) infections \\
\hline 2) malignancies \\
\hline 3) rheumatic diseases \\
Diagnosis: 5 criteria, at least 2 major \\
\hline
\end{tabular}

agnosis of AOSD was confirmed and glucocorticosteroids in pulse therapy (methylprednisolone $500 \mathrm{mg}$, i.v., on three successive days) were initiated again, together with immunosuppressive treatment with methotrexate at a dose of $15 \mathrm{mg}$ per week and indomethacin at 75 mg per day. A partial therapeutic effect was achieved by resolving joint pain and fever, with a partial drop in inflammatory markers.

After one month of therapy, despite adhering to current medical recommendations and regular treatment, the patient still presented clinical symptoms and significant deviations in auxiliary tests. The laboratory test results were comparable to test results from before the therapy was initiated. Due to lack of improvement and because no disease remission was achieved, it was decided to intensify anti-inflammatory treatment, by administering again methylprednisolone parenterally at a total dose of $3 \mathrm{~g}$ per treatment cycle. Because of severe anemia with very low Fe serum levels, iron was also administered parenterally. At the same time, the MTX dose was increased to $20 \mathrm{mg}$ per week. However, despite the applied treatment, the expected therapeutic effect was not achieved, and for some inflammatory markers the results deteriorated (Table III). Lack of significant response to treatment resulted in an urgent attempt to verify the diagnosis. The imaging scans were repeated, with deviations including widened mediastinum in the $X$-ray image, and lymph node packets in the computed scan of the chest (Fig. 1). Due to visibly increasing serum platelet levels and suspected essential thrombocythe- 
Table III. Laboratory tests values in successive weeks of treatment

\begin{tabular}{|lccccc|}
\hline Parameter & On admission & 1 week & 4 weeks & 6 weeks & Norm \\
\hline ESR $[\mathrm{mm} / \mathrm{h}]$ & 119 & 28 & 118 & 115 & $3-15$ \\
\hline CRP $[\mathrm{mg} / \mathrm{l}]$ & 303 & 173 & 299 & 178 & $0-5$ \\
\hline WBC $\left[10^{3} / \mathrm{mm}^{3}\right]$ & 18 & 14 & 21 & 11 & $4-10$ \\
\hline PLT $\left[10^{3} / \mathrm{mm}^{3}\right]$ & 819 & 550 & 1161 & 661 & $140-440$ \\
\hline Hemoglobin $[\mathrm{mmol} / \mathrm{l}]$ & 5,3 & 5,2 & 4,5 & 4,0 & $7.4-9.9$ \\
\hline Ferritin $[\mathrm{ng} / \mathrm{ml}]$ & 1952 & 1200 & 632 & 4358 & $13-150$ \\
\hline$\gamma$-globulin $[\%]$ & 27.3 & 25 & 29 & 26 & $11-21$ \\
\hline
\end{tabular}

mia, a test for mutation of the tyrosine kinase gene, JAK2, occurring in myeloproliferative disorders was performed. However, this diagnosis was not confirmed. At the same time, due to significant anemia, it was decided to test for sTfR (soluble transferrin receptor), helpful in differential diagnosis of various types of anemia. Despite the substitutive treatment according to calculated daily demand, increased STfR levels were found, which indicated anemia of chronic-inflammatory diseases, rather than an actual iron deficiency.

Similarly, no presence of the fusion gene BCR/ABL was demonstrated with the FISH (fluorescence in situ hybridization) method. This gene is responsible for tyrosine kinase production stimulating incorrect proliferation of bone marrow stem cells, occurring in chronic myelogenous leukemia. Only slightly increased alkaline phosphatase activity in the peripheral blood granulocytes (FAG) was found. During further hematologic diagnosis, peripheral blood cytometry was performed, as well as bone marrow trepanobiopsy with its evaluation in flow cytometry. Virological assays as well as blood and urine culture tests were also performed. None of

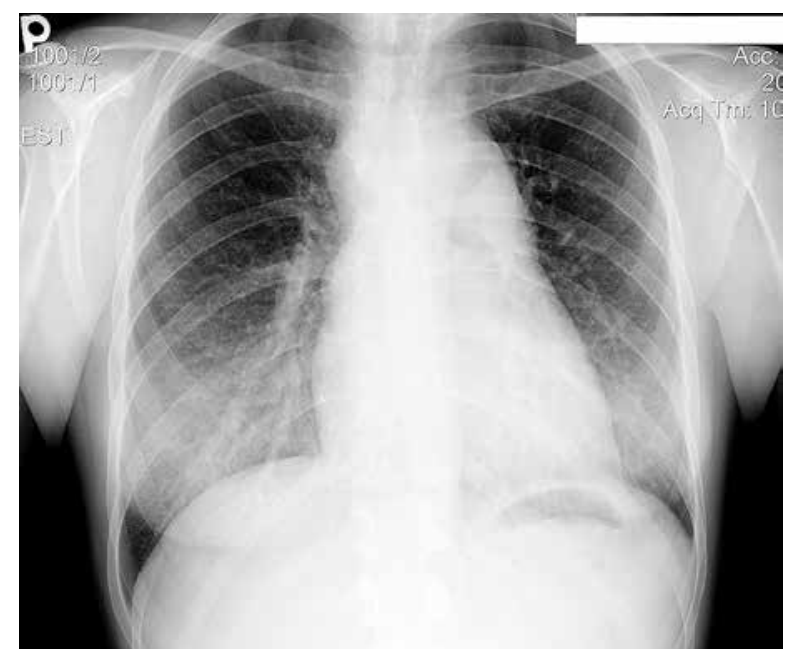

Fig. 1. Chest X-ray anteroposterior - extended shadow caused by enlarged mediastinal lymph nodes. the tests listed above confirmed or excluded the diagnosis; therefore, video-assisted thoracoscopic surgery with collection of the lymph node section was performed for the final diagnosis. On the basis of the histopathological test results of the lymph node, the patient was finally diagnosed with Hodgkin lymphoma and transferred to a hematology centre for further treatment.

\section{Comment}

Adult-onset Still's disease is a rare syndrome, which is poorly recognizable in daily clinical practice, as it is difficult to diagnose, which significantly prolongs the time until effective therapy is initiated [1,2]. This disease was observed and described in children by Sir George Frederick Still in the $19^{\text {th }}$ century, and it is now considered to be one of the systemic forms of juvenile idiopathic arthritis (JIA) [1]. Adult-onset Still's disease was described by Eric Bywaters in the 1970s.

The disease can be asymptomatic for a long time, or it may present a full range of classic symptoms supported with laboratory and imaging study results [3-6]. Often a long time can elapse before a patient is consulted by a rheumatologist. Adult-onset Still disease can be masked by other diseases, in particular by infections or hematologic neoplasms treated without the expected therapeutic effect. The first symptoms are usually observed in young people, before 35 years of age. Possible risk factors include viral or bacterial infections, but the genetic predisposition is also important [1-3].

The disease presentation includes systemic symptoms, often preceded by laryngitis, which was not observed in the interview in the described patient. A predominant sign found in $90 \%$ of patients is a hectic fever $[2,4]$ with a maculopapular rash appearing at its height. In the patient the high fever was not accompanied by skin lesions. The so-called salmon-colored rash is quite specific though often difficult to observe and transient. Other symptoms are often non-specific. They include: loss of body weight, asthenia, muscle ache, enlargement of lymph nodes, liver, or spleen, pleuritis and pericarditis, as well as myocarditis [5]. In the described patient, 
asthenia and muscle aches predominated at the beginning, but lymphadenopathy was not observed, and the observed organ damage indicators included increased liver tests and fluid in the pericardium. Symptoms of arthritis and cervical spine involvement are present in nearly all patients with adult-onset Still disease. However, they do not have to be a leading symptom, as in the reported case. Due to non-specific clinical symptoms and the laboratory presentation, diagnosis of adult-onset Still's disease is made only after exclusion of other diseases, such as diseases of infectious etiology, hematologic neoplasms with particular focus on all lymphomas, or other systemic diseases of connective tissue. Before treatment was initiated the reported patient was diagnosed at the hematology unit, and at that disease stage no signs of proliferative process were observed.

Adult-onset Still's disease can be diagnosed based on the classification criteria established by Yamaguchi et al. This approach was used in the reported case with clinical symptoms and results of the laboratory tests considered [6]. Analyzing the above criteria, the patient met 3 out of 4 main criteria and 2 out of 4 minor criteria, where 5 criteria in total (minimum of 2 major ones) are sufficient for the diagnosis.

It should be emphasized that unfortunately there is no specific diagnostic test for AOSD. However, the presence of a high serum ferritin level is important [7], as some authors consider its increase $>1000 \mu \mathrm{g} / \mathrm{l}$ as a diagnostic marker for this disease. It is also used to monitor treatment effectiveness. In the reported case the ferritin level was significantly increased, but contrary to expectations, its value did not decrease but remained increased during the applied treatment. Sometimes more precise determination of the glycosylated ferritin fraction is used in the in-depth diagnostics. Its level below $20 \%$ belongs to diagnostic criteria according to Fautrel et al. (Table IV) [2, 8]. Unfortunately, this test was not accessible in the reported patient.

Although uniform therapeutic standards for treatment of AOSD are not established, in general therapy includes use of nonsteroidal anti-inflammatory drugs, corticosteroids, and immunosuppressive drugs, mainly methotrexate [9] and in specific cases also biological therapy (anti-TNF- $\alpha$, anti-IL-1, or anti-IL-6) [3, 8-11]. A good and quick therapeutic response particularly to high CS doses usually confirms the correct diagnosis. Unfortunately, in the patient described, the high inflammatory parameters, anemia and thrombocythaemia persisted despite high-dose intravenous corticosteroid therapy followed by oral therapy and immunosuppression, and additionally later lymph node packets appeared in the mediastinum. It should be noted that the mediastinal lymphadenopathy can also be a part of the
Table IV. Criteria of adult-onset Still's disease (Fautrel B. et al., 2001)

\begin{tabular}{|l|}
\hline \multicolumn{1}{|c|}{ Major criteria } \\
\hline Spiking fever $\geq 39^{\circ} \mathrm{C}$ \\
\hline Arthralgia \\
\hline Transient erythema \\
\hline Pharyngitis \\
\hline Polymorphonuclear $\geq 80 \%$ \\
\hline Glycosylated ferritin $\leq 20 \%$ \\
\hline \\
\hline Maculopapular rash \\
\hline White blood cells $\geq 10000 / \mu 1$ \\
\hline
\end{tabular}

Diagnosis: 4 major criteria or 3 major + 2 minor

clinical presentation of other rheumatological diseases or bacterial and viral infections. However, lack of a therapeutic effect and disease progress prompted repeated enhanced haematological diagnostic tests, of which only the histopathological examination of the lymph node resulted in the final diagnosis of Hodgkin lymphoma. The patient was entrusted to the care of hematologists, but her later whereabouts remain unknown.

When diagnosing and managing treatment of AOSD, clinical and diagnostic caution must be observed, particularly in cases without a spectacular therapeutic effect. Clinical difficulties may include complications emerging from the progressing disease, and also from treatment implemented for co-infections, which usually blur the clinical presentation. However, sometimes the course of disease and therapy forces a rheumatologist to review the diagnosis. We should also keep in mind that diagnosis of one disease does not exclude a concurrent one. Thorough analysis by an experienced clinician also requires a certain reserve when approaching all auxiliary tests, which will never replace a general medical approach. The psychological aspects including fear for health and life, frustration with severe chronic disease and episodes of depression should also be considered, as they sometimes contribute to treatment failure. Therefore, in adult-onset disease, diagnostic concerns, therapy, disease course and prognosis are important issues in rheumatological practice.

The authors declare no conflict of interest.

\section{References}

1. Efthimiou P, Paik PK, Bielory L. Diagnosis and management of adult onset Still's disease. Ann Rheum Dis 2006; 65: 564-572. 
2. Leszczyński P, Pawlak-Buś K, Szafrański T. Adult onset Still's disease as the cause of fever of unknown etiology. Post Nauk Med 2012; 2: 120-127.

3. Van de Laar M, Vonkeman H, Bresnihan B. Miscellaneous inflammatory arthritides: adult Still's disease and sarcoidosis. In: Eular Compendium on Rheumatic Diseases. Bijlsma WJ (ed.). 1st Edition. BMJ Publishing Group, London 2009; 149154.

4. Cristin JC, Martinem-Banos D, Alcocer-Varela J. Adult-onset Still disease as the cause of ever of unknown origin. Medicine (Baltimore) 2005; 84: 331-337.

5. Miczke A, Waśniewski M, Straburzyńska-Migaj E, et al. Myocarditis - the first symptom of adult Still's disease. Kardiol Pol 2009; 67: 884-886.

6. Mueller R, Sheriff A. Scoring Adult Onset Still's Disease. Rheumatol 2010; 37: 2203-2204.

7. Mehta B, Efhimiou P. Ferritin in Adult-Onset Still's Disease: Just a Useful Innocent Bystander. Int J Inflamm 2012; 2012: 298405.

8. Kontzias A, Schett G. Adult-onset Still's disease: pathogenesis, clinical manifestations and therapeutic advances. Drugs 2008; 68: 319-337.

9. Manger B, Rech J, Schett G. Use of methotrexate in adult-onset Still's disease. Clin Exp Rheumatol 2010; 28 (Suppl 61): 168-171.

10. Fleischmann R, Iqbal I, Nandeshwar P, et al. Safety and efficacy of diseasemodifying anti-rheumatic agents: focus on the benefits and risks of etanercept. Drug Saf 2002; 25: 173-197.

11. Thonhofer R, Hiller M, Just $\mathrm{H}$, et al. Treatment of refractory Adult-onset Still's disease with tocilizumab; report of two cases and review of the literature. Rheum Int 2011; 31: 1653-1656. 